\title{
Memory loss after stroke
}

Alberto Maud, MD

Memory loss commonly occurs as a result of the loss of nerve cells in the brain. When memory loss is so severe that it interferes with normal daily functioning, it is called dementia. People with dementia may have difficulty learning new things or remembering names of people they just met. They may get lost in places that were previously very familiar or have trouble finding words. The term "mild cognitive impairment" is used for a common condition in elderly people in which the memory problem is usually mild and does not interfere with normal daily activities. Most people with mild cognitive impairment do not develop a severe memory problem or end up developing dementia. There are many things that can cause dementia. One of these is Alzheimer disease (AD), the most common cause of dementia in the elderly. Another is called vascular dementia, caused by brain damage due to strokes. More information about causes of dementia can be found on the next page.

In this issue of Neurology, O'Brien and colleagues report the results of a study in elderly people evaluating the risk of developing dementia after a person has had a stroke (Gamaldo A, Moghekar A, Kilada S, Resnick SM, Zonderman AB, O'Brien R. Effect of a clinical stroke on the risk of dementia in a prospective cohort. Neurology 2006;67:1363-1369). The study included 335 elderly subjects who were on average 75 years of age when they entered the study. None of them had a memory problem, stroke, or major brain disease in the past. Subjects were evaluated on a yearly basis for memory problems and for whether they had a stroke. Overall, subjects were followed in the study for an average of 10 years.

During the follow-up period, roughly half of the study subjects developed mild cognitive impairment. There were 36 subjects who had stroke. Of the group of subjects who developed a stroke, about two-thirds of them also developed dementia in the first year after the stroke. The majority of patients with dementia after stroke had mild cognitive impairment prior to the occurrence of the stroke. Most of the subjects with mild cognitive impairment who did not have a stroke ever developed severe memory problems over the course of the study and some even showed signs of improvement.

There were 157 patients who died during the follow-up period. Autopsies were performed in 22 patients who had had a stroke and 108 who had not. Of the 108 subjects who had not had a stroke during follow-up, 26 of them were found to have brain damage caused by a stroke that did not cause any symptoms during life. These autopsy studies of the brain showed that people with strokes found only at autopsy were more likely to have mild cognitive impairment than subjects who did not have these abnormalities. The researchers also found brain abnormalities that are also seen in patients with AD in half of the subjects who died. These AD changes seemed to be a risk factor for cognitive impairment in these elderly subjects.

\section{What did we learn from this study?}

It seems clear that mild cognitive impairment is a common problem in elderly people. In most people, the memory problems do not progress and may even improve with time. However, in people with mild cognitive impairment who also have a stroke, there is a much greater chance for developing dementia. The fact that almost half of the subjects who died had brain abnormalities also seen in people with AD suggests that these abnormalities may be a cause of mild cognitive impairment in the elderly.

Why is this important for me? While mild cognitive impairment was very common in this very elderly population, as long as subjects did not also have a stroke, their memory problems remained mild. This suggests that it is very important for elderly people to lower their risk factors for stroke as much as possible to help prevent dementia. This includes controlling high blood pressure, diabetes, and high cholesterol levels, remaining physically active, and not smoking cigarettes. 
Stroke is a "brain attack" commonly caused either by a sudden loss of blood flow to the brain or by a bleeding inside the head. Eighty percent of all strokes are called ischemic due to loss of blood flow. About $20 \%$ of strokes are called hemorrhagic due to bleeding.

Large ischemic strokes are usually caused by narrowing of the large arteries in the neck and brain due to atherosclerosis. If the clot forms in the neck vessels, pieces can break off and block the brain vessels. Clots may also arise from the heart and travel to the brain vessels where they become lodged. Large ischemic strokes usually give symptoms such as muscle weakness, loss of feeling, speech and vision problems, double vision, headache, unsteadiness, sleepiness, and sometimes death. People with uncontrolled high blood pressure and diabetes often have small ischemic strokes that involve very small arteries in the brain. Small ischemic strokes may not cause any symptoms. It is not unusual to find these asymptomatic, or "silent" strokes on brain scans that are done for other reasons.

It is estimated that approximately one third of stroke victims will de- velop memory problems and serious difficulties in other aspects of performing daily activities. The memory problems can be so severe that they interfere with normal functioning and are then called dementia. This is more common as people get older. When dementia occurs after a stroke and no other cause can be found it is called vascular dementia.

Both large strokes strategically located in certain areas of the brain or multiple small strokes can result in vascular dementia. Certain features like old age, prior memory problems, a history of several strokes, or a stroke located in the left side of the brain all seem to increase the likelihood of dementia in the first year after stroke.

The symptoms of vascular dementia in addition to the memory loss include slow movement, slow thinking, lack of attention, and lack of an ability to do simple tasks. These symptoms are sometimes hard to differentiate from those produced by $A D$, the most common cause of dementia in the elderly. Symptoms of dementia after stroke can also be hidden by other more obvious stroke manifestations like paralysis, blindness, or lack of awareness. Another problem in recognizing the symptoms of dementia after stroke is that these symptoms can be confused with depression, which is quite common after stroke.

\section{Can we prevent memory loss after stroke?}

The best way to prevent dementia after stroke is to avoid having a stroke. Just controlling high blood pressure can markedly reduce the risk of having a stroke. Having regular check-ups, exercising, eating healthily, keeping a normal cholesterol and blood sugar level, and quitting smoking can dramatically reduce the risk of stroke as well.

\section{How do we treat memory loss that occurs after a stroke? There is no specific medical treat- ment to help reverse the memory loss that occurs after a stroke. Neu- rologists sometimes prescribe medi- cations approved for AD for people with vascular dementia, but we do not yet have studies to address the usefulness of these medications in patients with vascular dementia.}

\section{For more information}

American Academy of Neurology www.aan.com

American Stroke Association, a division of the American Heart Association www.strokeassociation.org 


\title{
Neurology
}

\author{
Memory loss after stroke \\ Alberto Maud \\ Neurology 2006;67;E14-E15 \\ DOI 10.1212/01.wnl.0000244752.95386.de
}

\section{This information is current as of October 23, 2006}

\section{Updated Information \&} Services

\section{Supplementary Material}

Permissions \& Licensing

Reprints including high resolution figures, can be found at: http://n.neurology.org/content/67/8/E14.full

Supplementary material can be found at: http://n.neurology.org/content/suppl/2007/11/08/67.8.E14.DC1

Information about reproducing this article in parts (figures,tables) or in its entirety can be found online at:

http://www.neurology.org/about/about_the_journal\#permissions

Information about ordering reprints can be found online: http://n.neurology.org/subscribers/advertise

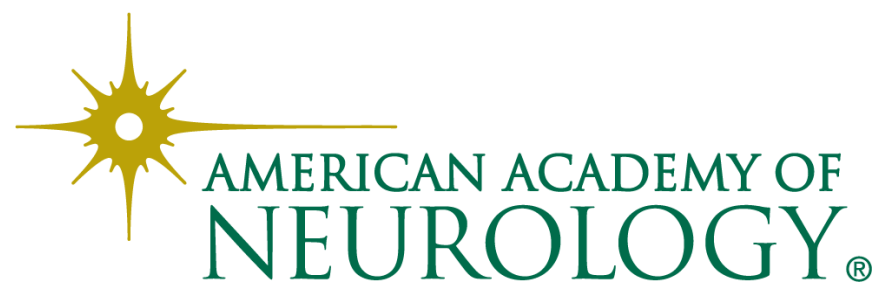

